\title{
Variability and Maintenance of Turbulence in the Very Stable Boundary Layer
}

\author{
Larry Mahrt
}

Received: 31 May 2009 / Accepted: 14 December 2009 / Published online: 30 December 2009

(C) The Author(s) 2009. This article is published with open access at Springerlink.com

\begin{abstract}
The relationship of turbulence quantities to mean flow quantities, such as the Richardson number, degenerates substantially for strong stability, at least in those studies that do not place restrictions on minimum turbulence or non-stationarity. This study examines the large variability of the turbulence for very stable conditions by analyzing four months of turbulence data from a site with short grass. Brief comparisons are made with three additional sites, one over short grass on flat terrain and two with tall vegetation in complex terrain. For very stable conditions, any dependence of the turbulence quantities on the mean wind speed or bulk Richardson number becomes masked by large scatter, as found in some previous studies. The large variability of the turbulence quantities is due to random variations and other physical influences not represented by the bulk Richardson number. There is no critical Richardson number above which the turbulence vanishes. For very stable conditions, the record-averaged vertical velocity variance and the drag coefficient increase with the strength of the submeso motions (wave motions, solitary waves, horizontal modes and numerous more complex signatures). The submeso motions are on time scales of minutes and not normally considered part of the mean flow. The generation of turbulence by such unpredictable motions appears to preclude universal similarity theory for predicting the surface stress for very stable conditions. Large variation of the stress direction with respect to the wind direction for the very stable regime is also examined. Needed additional work is noted.
\end{abstract}

Keywords Intermittency $\cdot$ Nocturnal boundary layer $\cdot$ Richardson number $\cdot$ Stable boundary layer $\cdot$ Submeso

\section{Introduction}

The turbulent flux at a given point in space and time in the stable boundary layer may deviate substantially from that predicted from the mean flow. For example, for a given mean state,

L. Mahrt ( $\square)$

Oregon State University, Corvallis, OR 97331, USA

e-mail: mahrt@nwra.com 
as often represented by the Richardson number, the surface drag coefficient is characterized by a broad probability distribution of values. This variability increases with increasing stability. The variation of turbulence quantities for a given mean state has received much less attention than the prediction of averaged turbulence conditions and is not included in parameterization schemes. However, this variation is of both theoretical and practical interest. For example, in a weak mixing environment, an isolated event of stronger turbulent mixing may have only a modest influence on time-averaged quantities, but may have a critical influence on whether the instantaneous surface temperature decreases below a critical value (such as freezing) or whether the concentration of a harmful contaminant exceeds a threshold value where damage ensues. From a more general point of view, a random process has zero net influence on a system only if that system is linear.

Turbulence quantities, such as dissipation, may vary locally by orders of magnitude (Muschinski et al. 2004). The variability of the turbulence is sometimes posed in terms of intermittency, although the definition of intermittency varies between studies, depending on scale and depending on the method used to detect intermittency. The intermittency in the stable atmospheric boundary layer is not an on-off process. The turbulence may be generated by instability events, but the amplitudes of such bursts of activity vary and the turbulence events at a fixed measurement point are observed in various stages of decay such that the turbulence intensity takes on a continuous distribution of values. The simplest measure of intermittency is kurtosis, often applied to velocity or temperature differences (Mahrt and Howell 1994; Viana et al. 2008). Cava and Katul (2009) explored fine-scale intermittency in terms of clustering properties of sign switches of scalar fluctuations and examined potential scaling laws.

Many techniques define intermittency in terms of turbulence quantities exceeding certain thresholds (Blackwelder and Kaplan 1976; Nappo 1991; Katul et al. 1994). Howell and Sun (1999) define intermittency in terms of the fraction of the record required to account for a certain fraction of the total flux, which emphasizes the larger transporting eddies. Following Howell and Sun (1999), a number of studies, including Coulter and Doran (2002), Acevedo et al. (2006), Basu et al. (2006), have documented the flux intermittency for a variety of situations in the stable boundary layer. More complex event-finding procedures were applied in Nakamura and Mahrt (2005), who showed that the horizontal scale of intermittent mixing events was often on the order of $100 \mathrm{~m}$ or less. Salmond (2005) examined the intermittency in terms of the Haar decomposition of the vertical velocity fluctuations, through partitioning the signal into turbulent and non-turbulent components and separately analyzing the spectral behaviour for both components. While the intermittency is generally observed to increase with stability, the turbulence bursts may be rare for the most stable cases. In this "radiation" regime (Van de Wiel et al. 2003), the vertical divergence of the very weak turbulence heat flux may be less important than the radiative flux divergence. Intermittency of the dissipation can be statistically related to fine-scale variations of vertical temperature gradients, providing local similarity (Sorbjan and Balsley 2008).

The intermittency is driven both by random variations (Yaglom 1973), characteristic of turbulence with a stationary mean flow, and by non-stationarity due to motions on time scales just larger than the turbulence. These motions are sometimes referred to as submeso motions (Mestayer and Anquetin 1995; Anquetin et al. 1998; Belušić and Mahrt 2008). Such motions are always present and cause the flow to be significantly non-stationary when the large-scale flow is weak. This non-stationarity is often visualized in terms of large variations of the wind direction on time scales of tens of minutes (Hanna 1986; Etling 1990; Anfossi et al. 2005) and large abrupt shifts of wind direction on time scales of a minute or less (Mahrt 2008). In the stable boundary layer, non-stationary submeso motions on time scales 
of minutes or tens of minutes include wave-like phenomena (e.g., Nappo 2002; Fritts et al. 2003), two-dimensional modes, non-stationary drainage flows (Doran and Horst 1981), microfronts, propagating density currents and solitary waves (Sun et al. 2002, 2004; Anderson 2003; Terradellas et al. 2005) and a variety of other more complex modes (Mahrt 2007a). The submeso energy is generally greater in complex terrain except for well-defined slope flows (Vickers and Mahrt 2007). Universal spectra in the submeso range are not observed, but rather the energy distribution varies substantially between sites (Vickers and Mahrt 2007; Mahrt 2009).

Weak-wind flows near the surface are typically a complex mixture of different modes. As a result, the generation of turbulence by specific submeso modes has been difficult to isolate. Detailed information is available in simpler numerical settings with well-defined gravity waves (e.g. Fritts et al. 2009). The more general influence of non-stationary submeso motions on the turbulence cannot be posed as a random process related to a single forcing and population of data nor can equilibrium turbulence be assumed. Since weak-wind, very-stable boundary layers are almost always significantly non-stationary, we must proceed without the formal support of the concept of an ensemble average. The turbulence and the submeso "mean flow" continuously interact, corresponding to a non-stationary flow (Derbyshire 1995; Van de Wiel et al. 2002). Quantification of non-stationarity in the boundary layer is discussed by Panofsky and Dutton (1984) and Andreas et al. (2008). Influences of the non-stationary submeso motions (Mahrt 2007b) leads to turbulence in the weak-wind stable regime that is more complex than in laboratory flows (e.g. Ohya et al. 2008).

As a partial result of the shear generation of turbulence by submeso motions, the turbulence does not vanish at very large Richardson number. Maintenance of turbulence at large Richardson numbers and the lack of a critical Richardson number has been discussed in detail by Galperin et al. (2007) and Zilitinkevich et al. (2007) and has been noted in numerous observational studies (for a recent example, see Luhar et al. 2009). While turbulence can become extremely weak, it does not completely vanish near the surface (Mahrt and Vickers 2006).

The variability of scaled turbulence quantities, such as the non-dimensional gradients, is not well predicted by the Richardson number in very stable conditions (e.g., Grachev et al. 2005; Luhar et al. 2009), even after averaging over 30 minute or one hour and discarding data through conditions on maximum nonstationarity, maximum stability or minimum turbulence. Yagüe et al. (2006, their Figures 10-11) explicitly show the near independence of $\phi_{m}$ and $\phi_{h}$ from the gradient Richardson number for large Richardson number and quantify the increasing scatter with increasing Richardson number. Their results suggest that other processes, not represented by the Richardson number, might be controlling the variability of the momentum flux, such as "internal wave bursts." Since such waves and other submeso motions can generate turbulence through shear instability, the unpredictability of the submeso motions leads to a certain degree of unpredictability of the turbulence in weak-wind very stable conditions. The potential generation of turbulence by submeso shear is of considerable interest, since boundary-layer submeso motions are generally not included in numerical models because of the lack of resolution and/or lack of physics.

The impact of such non-stationary motions may be quite different for momentum as compared to heat (Mahrt 2007b) resulting in a stability dependent eddy Prandtl number (Grachev et al. 2007; Anderson 2009). The momentum flux may vary more than the heat flux (Basu et al. 2006).

Partly motivated by the studies of Yagüe et al. (2006) and others, the present study examines the variability of the turbulence for very stable conditions in more detail. Since the turbulence energy is generally weak for very stable conditions and averaged quantities are often 
dominated by a limited number of turbulence events, even if poorly defined, the skewness of the turbulence becomes a simple useful indicator of variability, although other measures of variability will be used. We do not eliminate any data by imposing conditions on non-stationarity, erratic wind profiles or minimum turbulence nor impose any other conditions to reduce scatter. Instead, this study focuses on the behaviour of the scatter in the relationships between the turbulence and the mean flow and attempts to determine the role of submeso motions in maintaining turbulence at large Richardson number.

\section{Data}

As the primary dataset (Table 1), we analyze four months of nocturnal eddy-correlation data from Fluxes Over Snow-covered Surfaces II (FLOSSII) carried out from 1 December 2002 to 31 March 2003 in the North Park Basin of north-west Colorado, U.S.A. (Mahrt and Vickers 2006). The 30-m tower provides seven levels of eddy-correlation data over a grass surface, sometimes partially or completely snow covered. While the site is surrounded by complex terrain, the surface within a few kilometres of the site is relatively flat. Although this site is generally windy, the four months of data provide a significant number of weak-wind cases.

We briefly analyze one month of eddy-correlation data from a 60-m tower with seven levels of eddy-correlation data over a grassland in south central Kansas, U.S.A. in CASES-99 (Cooperative Atmospheric Surface Exchange Study, Poulos et al. 2002; Sun et al. 2002). The CASES-99 dataset includes a limited amount of very stable data, but has become the standard stable boundary-layer dataset. One year of data from two eddy-correlation sites in central Oregon, U.S.A. is also analyzed. One dataset was collected over a $15-\mathrm{m}$ mature ponderosa pine (Schwarz et al. 2004) and one over a largely burned ponderosa pine (Burn site, Mahrt 2006).

Fluxes are computed following the approach of Vickers and Mahrt (2006), which chooses the averaging length for the definition of perturbations, $\tau$, in terms of the cospectra, as in the OGIVE technique (e.g., Friehe et al. 1991). In this regard, the turbulence is defined as the small-scale part of the cospectra where the heat flux is systematically gradient. The weakwind very stable cases sometimes correspond to ambiguous cospectra, in which case the method is of unknown accuracy. A detailed case study approach based on wavelet analysis (Cuxart et al. 2002; Viana et al. 2008) provides more insight into this problem, although a corresponding systematic method for calculation of fluxes from large datasets has not been developed.

Table 1 For very stable conditions $(R b>1)$, the datasets (first column), the number of hourly records (no.), the site-averaged $\sigma_{w}\left(\mathrm{~m} \mathrm{~s}^{-1}\right), u_{*}$ based on site-averaged values of the momentum flux $\left(\mathrm{m} \mathrm{s}^{-1}\right)$, the site-averaged submeso velocity scale $\left(v_{\text {smeso }}, \mathrm{m} \mathrm{s}^{-1}\right)$, the velocity aspect ratio for the submeso flow $\left(V A R_{\text {smeso }}\right)$, the velocity aspect ratio for the turbulence $\left(V A R_{t u r b}\right)$ and the correlation between $\sigma_{w}$ and $v_{s m e s o}$ (corr.)

\begin{tabular}{lrllllll}
\hline Data & No. & $\sigma_{w}$ & $u_{*}$ & $v_{\text {smeso }}$ & VAR $_{\text {Smeso }}$ & $V_{\text {turb }}$ & Corr. \\
\hline FLOSSII & 176 & 0.052 & 0.028 & 0.77 & 0.0006 & 0.09 & 0.67 \\
CASES-99 & 12 & 0.048 & 0.036 & 0.68 & 0.0010 & 0.05 & 0.76 \\
Pine & 565 & 0.087 & 0.039 & 0.61 & 0.0052 & 0.40 & 0.42 \\
Burn & 522 & 0.087 & 0.041 & 0.42 & 0.0076 & 0.23 & 0.38 \\
\hline
\end{tabular}

For the Pine and Burn sites, the data correspond to $z / L>1$ for qualitative comparison 


\section{Averaging and Intermittency}

A given variable, $\phi$, is initially partitioned as

$$
\phi^{\prime}=\phi-[\phi]_{\tau}
$$

where the operator []$_{\tau}$ averages over time interval $\tau$ to separate the turbulence from the larger-scale flow. $\tau$ must be a large enough scale to remove the largest turbulent eddies. The value of $\tau$ (Sect. 2) generally decreases with increasing stability and may become smaller than ten seconds for individual records.

The covariances are computed from the product of perturbation quantities from Eq. 1 . The covariances are then averaged over a short averaging time, $\tau_{s}$, to define a local value of the covariance

$$
\overline{w^{\prime} \phi^{\prime}}
$$

where the overbar corresponds to averaging over the short averaging time $\tau_{s}$. The short averaging time will be nominally chosen as one minute, which is usually comparable to, or larger than, the variable averaging time for defining the perturbations. Averages over $\tau_{s}$ will be used to study the short-term variability of the flux. We do not choose $\tau_{s}$ to be the record-dependent $\tau$ since a variable averaging time complicates the interpretation of subsequent analyses.

To reduce sampling problems, turbulence covariances are normally averaged over a longer time scale ("record length"), sometimes referred to as the flux-averaging time scale, $\tau_{F}$. In the present study, $\tau_{F}$ is arbitrarily chosen as one hour. As an example, the vertical turbulent flux of $\phi$ is then written as

$$
F=\left[\overline{w^{\prime} \phi^{\prime}}\right]=\left[w^{\prime} \phi^{\prime}\right]
$$

where the brackets correspond to averaging over the long time scale, $\tau_{F}$, and the turbulent quantities are defined as in Eq. 1. Since all averages are simple unweighted averages, the overbar becomes redundant after averaging over $\tau_{F}$, provided that $\tau_{F}$ is an integer multiple of $\tau_{s}$. Our study also uses the standard deviation of the vertical velocity for the record

$$
\sigma_{w}=\left[w^{\prime} w^{\prime}\right]^{0.5}
$$

as a general measure of the turbulence strength. The strength of the horizontal velocity fluctuations is more sensitive to the choice of averaging time scale compared to the vertical velocity fluctuations.

To represent the restriction of vertical motions by the stratification and presence of the ground surface, we evaluate the velocity aspect ratio for turbulence, defined for each 1-hour record as

$$
V A R_{t u r b} \equiv \frac{\sqrt{2}\left[w^{\prime} w^{\prime}\right]}{\left(\left[u^{\prime} u^{\prime}\right]^{2}+\left[v^{\prime} v^{\prime}\right]^{2}\right)^{0.5}} .
$$

The factor $\sqrt{2}$ in the numerator leads to $V A R_{\text {turb }}=1$ when all three variances are equal. The decrease of this ratio below unity represents the restraint of vertical motions by the stratification and ground surface.

\subsection{Within-Record Variability}

Our study examines both the variability of $\overline{w^{\prime} \phi^{\prime}}$ within records as well as the variability of $\left[w^{\prime} \phi^{\prime}\right]$ between records. We forego intermittency algorithms (Introduction) to represent the 
variability and instead compute the maximum 1- min value of $\overline{w^{\prime} \phi^{\prime}}$ scaled by the hourly value

$$
R \max \equiv \frac{\max \left(\overline{w^{\prime} \phi^{\prime}}\right)}{\left[w^{\prime} \phi^{\prime}\right]} .
$$

As a second simple measure, the skewness of the vertical velocity variance is chosen to represent the occasional mixing events embedded within the weak turbulence. The frequency distribution of positive definite quantities, such as the velocity variance or drag coefficient, is often characterized by positive skewness. We will also compute the scaled within-record standard deviation of the vertical velocity variance

$$
\sigma \overline{w^{\prime} w^{\prime}} /\left[w^{\prime} w^{\prime}\right]
$$

\subsection{Submeso Velocity Scale}

Submeso quantities are defined as the deviation of the short sub-record (1-min) averages from the record average

$$
\phi^{*}=\bar{\phi}-[\phi]
$$

where the overbar again refers to short time averages, here 1 minute, and the square bracket refers to averages over the record, here 1 hour. We use the submeso velocity scale (Eq. 6, Mahrt 2009) to represent the general strength of the submeso horizontal motions, defined as

$$
V_{\text {smeso }} \equiv\left[\left(u^{* 2}+v^{* 2}\right)^{0.5}\right] \text {. }
$$

$V_{\text {smeso }}$ also serves as a measure of non-stationarity within the record, since $V_{\text {smeso }}$ vanishes in the hypothetical limit of stationary flow. A velocity aspect ratio for submeso motions, $V A R_{\text {smeso }}$, is computed as

$$
V A R_{\text {smeso }} \equiv \frac{\sqrt{2}\left[w^{*} w^{*}\right]}{\left(\left[u^{*} u^{*}\right]^{2}+\left[v^{*} v^{*}\right]^{2}\right)^{0.5}} .
$$

\subsection{Stability}

The bulk Richardson number is used as a measure of overall stability that is less vulnerable to errors compared to the calculation of the gradient Richardson number for weak-wind conditions, where the vertical wind profiles can be complex. Use of $z / L$ relates turbulence quantities to other turbulence quantities, often with strong self correlation due to shared variables between scaled turbulence quantities and $z / L$. Anderson (2009) has recently developed a new technique for reducing the influence of self correlation, which allows better assessment of the validity of Monin-Obukhov similarity. However, the purpose of our study is to relate the turbulence to the stability of the mean flow defined independently of turbulence quantities. Use of $z / L$ automatically classifies significant turbulence as weak stability, even with strong stratification.

We compute the bulk Richardson number as

$$
R b=\left(g / \Theta_{o}\right) \frac{\left(\theta\left(z_{2}\right)-\theta\left(z_{1}\right)\right) z_{2}}{U\left(z_{2}\right)^{2}}
$$

where $\Theta_{o}$ is the record-averaged potential temperature, $U$ is the 1-hour vector average wind speed at an upper level $z_{2}$, and $z_{1}$ is the lower level chosen to be close enough to the ground that the height difference between the two levels is approximately $z_{2}$. The levels $z_{1}$ and $z_{2}$ straddle the level of the flux computation. For FLOSSII and CASES-99, the upper level is 
chosen to be $30 \mathrm{~m}$ while the lower level is selected as $1 \mathrm{~m}$ for FLOSSII and $1.5 \mathrm{~m}$ for CASES99. Fluxes are evaluated at $2 \mathrm{~m}$ for the FLOSSII data but at $5 \mathrm{~m}$ for CASES-99. For CASES-99 and FLOSSII, most nights are clear, in which case the wind speed, the vertical temperature difference and bulk Richardson number, are all strongly correlated. Strong (weak) wind conditions correspond to small (large) vertical temperature differences and small (large) $R b$.

Estimating the vertical temperature gradient over the tall vegetation in this study is problematic. The lowest temperature measurement level above the canopy is within the roughness sublayer and is presumably influenced by the proximity of the nearest underlying trees. For these sites, we use $(z-d) / L$ to represent stability instead of the bulk Richardson number, where $d$ is the displacement height. Even then, $(z-d) / L$ can be used only qualitatively since the displacement height is difficult to estimate. The tall vegetation sites are used only for qualitative comparisons with the short vegetation sites. In the ensuing relationships, we do not include the variance explained as a measure of statistical significance, since the relationships are often strongly non-linear, the distribution of variables is sometimes strongly non-Gaussian, and we do not offer empirical relationships for future application.

\section{Influence of Submeso Motions on Turbulence}

The following analyses concentrate on the nocturnal FLOSSII dataset; additional datasets are briefly considered in (Sect. 7). We first examine a simple relationship between the turbulence and mean flow, expressed here as the dependence of $\sigma_{w}$ on the wind speed. This investigation considers separately the stronger wind regime where the submeso velocity scale is less than the mean flow $V_{\text {smeso }}<V$ and the weaker wind regime where the submeso velocity scale is greater than the mean flow $V_{\text {smeso }}>V . \sigma_{w}$ depends systematically on $V$ for the more windy regime (Fig. 1, upper left, black points), but this dependence breaks down for the weaker wind regime (red points). For the weaker wind regime, $\sigma_{w}$ increases with increasing submeso velocity scale with an average rate of increase of about $0.1 V_{\text {smeso }}$ (Fig. 1, lower panel).

Fig. 1 The dependence of $\sigma_{w}$ on the speed of the large-scale flow, $V$, (upper-left panel) and the generalized velocity scale $V_{\text {gen }}$ (upper-right panel) where red dots correspond to $V_{\text {smeso }}>V$ and black dots correspond to $V_{\text {smeso }}<V$. The lower panel shows the dependence of $\sigma_{w}$ on $V_{\text {smeso }}$ for $V_{\text {smeso }}>V$
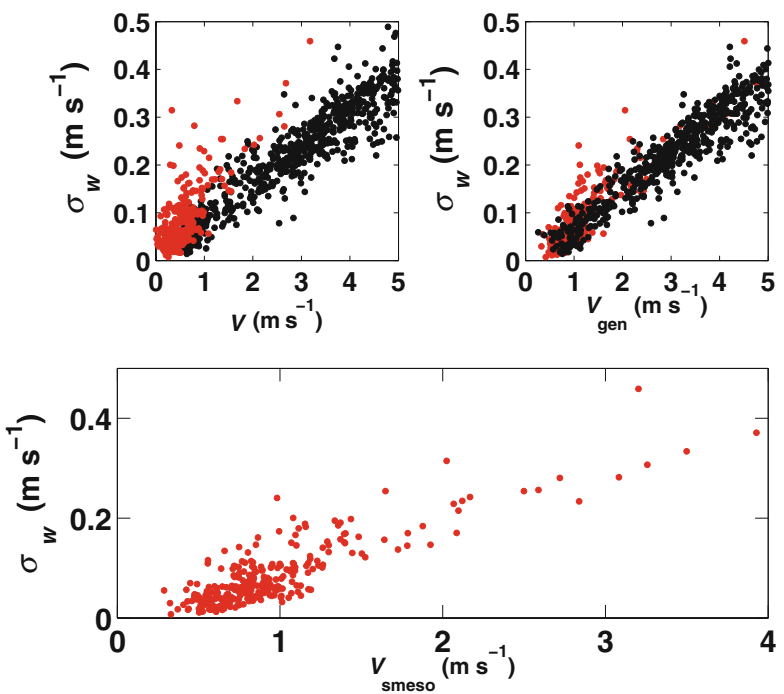
As a result of the dual influence of the mean and submeso velocity scales, simple similarity theory is not possible. However, $\sigma_{w}$ can be formulated in terms of a generalized velocity scale (Fig. 1, upper right), defined as

$$
V_{\text {gen }}=\left(V^{2}+V_{\text {smeso }}^{2}\right)^{0.5} \text {. }
$$

The scatter can be reduced further by including a coefficient in front of $V_{\text {smeso }}$ that is greater than unity, but optimization of empirical relationships is not the purpose of the present investigation. The generation of turbulence by the submeso flow is a non-stationary process and presumably quite different from the generation of the turbulence by the mean flow.

The submeso motions lead to a net increase of the record average turbulence, even though the submeso flow vector averages to zero over the record, by definition. An increase of total shear for parts of the record has a bigger influence than the decrease of shear for other parts of the record through triggering instabilities. In addition, the crosswind submeso shear increases the magnitude of the total vector shear, whether the crosswind shear is positive or negative.

\subsection{Friction Velocities}

While $u_{*}$ is often used as a scaling variable for $\sigma_{w}$, in this section we examine the relationship between $\sigma_{w}$ and $u_{*}$ as two co-varying turbulence quantities. The friction velocity is computed as

$$
u_{*}=\left(\left[w^{\prime} u^{\prime}\right]^{2}+\left[w^{\prime} v^{\prime}\right]^{2}\right)^{0.25}
$$

where the $x$-axis has been rotated into the direction of the mean flow and the covariances are defined by Eq. 3. The relationship between $\sigma_{w}$ (Eq. 4) and $u_{*}$ for significant turbulence is similar to previous studies (here, $\sigma_{w} \approx 1.2 u_{*}$ ). For weak turbulence, the relationship suffers more scatter and becomes sensitive to the inclusion of the crosswind momentum flux (Fig. 2, upper panel). Without the inclusion of the crosswind stress in Eq. 13, $u_{*}$ tends to vanish at a significantly faster rate than $\sigma_{w}$ (green plusses compared to red plusses in Fig. 2, upper panel). In the remainder of this paper, we include the crosswind stress in the calculation of $u_{*}$.

Fig. 2 Upper panel the relationship between $\sigma_{w}$ and $u_{*}$ for weak turbulence with (red) and without (green) the crosswind momentum flux $\left[w^{\prime} v^{\prime}\right]$. Lower panel the relationship between $\sigma_{w}$ and $u_{*}$ based on the usual hourly-averaged fluxes (red plusses) and the hourly average of 1-minute values of the friction velocity $\left[\left(u_{*}\right)_{1 \mathrm{~min}}\right]$ (black plusses), where the crosswind stress is included in both cases
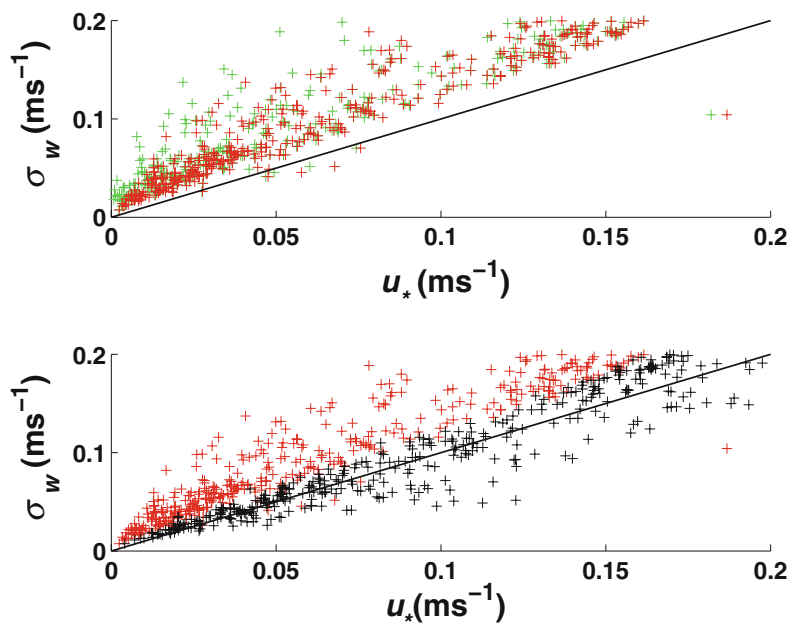
To include the variation of the turbulent momentum flux on submeso time scales, we also compute $\left(u_{*}\right)_{1 \text { min }}$ based on 1-min fluxes

$$
\left(u_{*}\right)_{1 \min }=\left(\left(\overline{w^{\prime} u^{\prime}}\right)^{2}+\left(\overline{w^{\prime} v^{\prime}}\right)^{2}\right)^{0.25}
$$

where the overbar again corresponds to 1 - min averages. These 1 - min values of $u_{*}$ are subsequently averaged over 1 hour to provide a modified version of the friction velocity, $\left[\left(u_{*}\right)_{1 \mathrm{~min}}\right]$. The values of $\left[\left(u_{*}\right)_{1 \mathrm{~min}}\right]$ (black plusses, lower panel Fig. 2) can be significantly larger than $u_{*}$ because the record-averaged magnitude of the 1-min crosswind stress is larger than the magnitude of the hourly-averaged crosswind stress. The hourly average of the crosswind momentum flux is reduced because of sign switches of the crosswind stress within the 1-hour record. The increased value of $u_{*}$, due to inclusion of the submeso (1-minute) variation of the turbulent momentum flux, leads to closer asymptotic behaviours between $u_{*}$ and $\sigma_{w}$ with vanishing turbulence. Evidently, submeso shear generation of turbulence increases the momentum flux; this effect is omitted based on the hourly-averaged momentum fluxes. This influence leads to the conflict between focussing on smaller averaging times to find a more suitable value of $u_{*}$ for comparison with $\sigma_{w}$, and using larger averaging times to increase the sample size.

\section{The Drag Coefficient}

\subsection{Dependence on the Bulk Richardson}

Scaling the momentum flux by the square of the wind speed provides the usual drag coefficient

$$
C_{d} \equiv \frac{u_{*}^{2}}{V^{2}}=f(R b)
$$

where $u_{*}$ is computed from 2-m record-averaged momentum fluxes using Eq. 13. Here, we choose $V=U$ where $U$ is the wind speed at the top of the layer used for evaluation of the bulk Richardson number, $30 \mathrm{~m}$ for FLOSSII. This leads to less scatter compared to the use of $2-\mathrm{m}$ winds. The $2-\mathrm{m}$ wind is more erratic than the $30-\mathrm{m}$ wind, while the $30-\mathrm{m}$ wind may be more representative of bulk shear instability.

Since both $C_{d}$ and the bulk Richardson number are inversely proportional to the mean wind speed squared, the above relationship is characterized by positive self correlation. This is opposite to the physically expected negative correlation; therefore, the observed negative correlation cannot be due to self correlation. Inclusion of the crosswind momentum flux formally makes the drag law incomplete since independent information on the stress direction is required (Sect. 8).

The drag coefficient decreases systematically with increasing bulk Richardson number up to a bulk Richardson number of order unity (Fig. 3). For bulk Richardson number $>1$, the drag coefficient appears to be independent of $R b$ (Fig. 3), with large scatter, including a dozen large offscale values. For example, occasional values of the drag coefficient occur that are much larger than any of the values for weak stability. These values occur because the speed of the mean wind is no longer an important velocity scale for $R b>1$; that is, extremely weak winds do not necessarily lead to extremely weak turbulence. The origin of the lack of importance of the mean wind speed is the poor relationship between the friction velocity and mean wind speed for $R b>1$ (Fig. 4, upper panel) in sharp contrast to the good relationship between the friction velocity and wind speed for $R b<1$ (Fig. 4, lower panel). For weak winds, the turbulence strength is more related to the submeso activity. 
Fig. 3 Hourly values of $C_{d}$ as a function of the bulk Richardson number. Twelve large values of $C_{d}$ are offscale
Fig. 4 The dependence of $u_{*}$ on the mean wind speed for $R b>1$ (upper panel) and for $R b<1$ (lower panel)
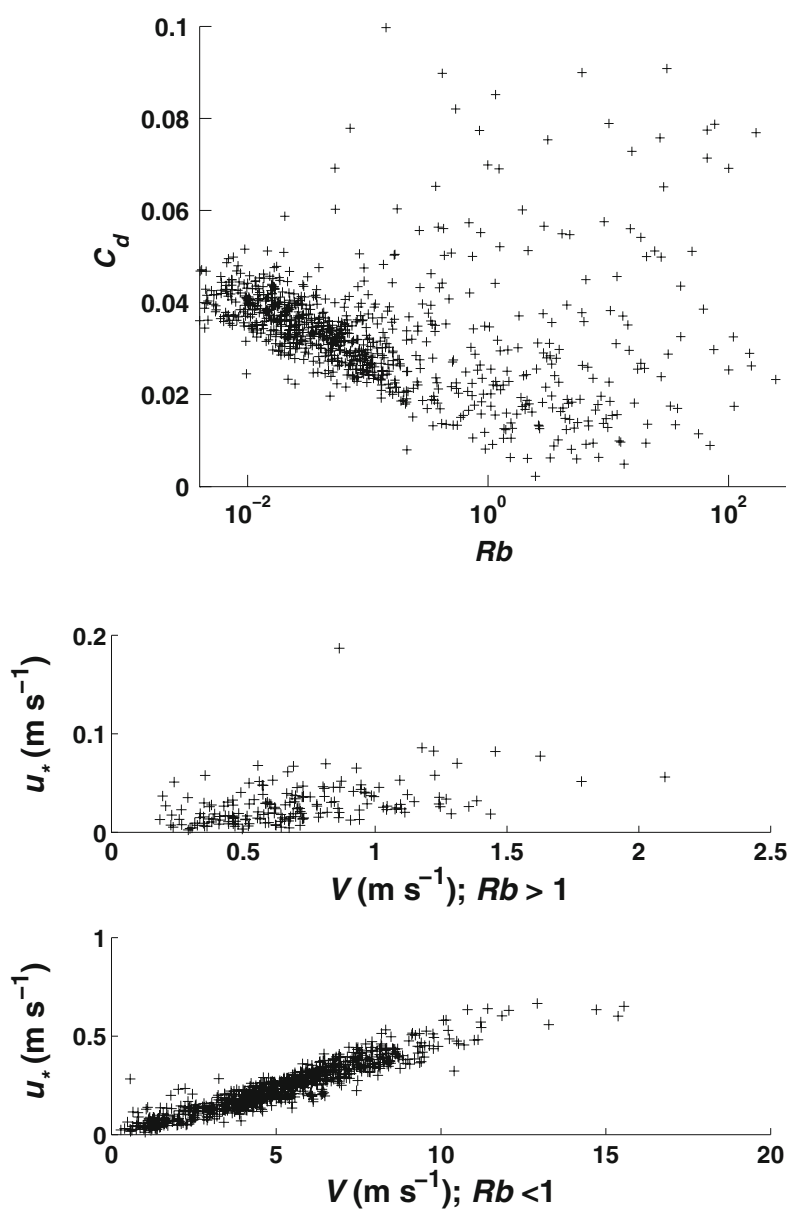

We casually refer to the regime of near independence of the bulk Richardson number (Fig. 3) as the very stable regime. We use $R b>1$ as a pragmatic identifier of this regime, although Fig. 3 suggests that this cut-off value could be smaller. The near independence from the bulk Richardson number indicates the absence of a critical value above which the turbulence decays. For reference, values of the bulk Richardson number are smaller than those of the gradient Richardson number so that $R b=1$ corresponds to a gradient Richardson number larger than 1, averaging about four times greater for weak winds. The large scatter in Fig. 3 has been reduced in some previous studies by imposing conditions on non-stationarity or minimum allowed turbulence quantities, or applying other techniques to eliminate outliers. Note that a few large values of the drag coefficient occur for smaller bulk Richardson numbers. These cases tend to occur with weak winds, weak stratification and significant submeso activity.

\subsection{Dependence on the Submeso Velocity Scale}

The large scatter in Fig. 3, particularly for $R b>1$, corresponds to a large range of turbulence intensities for a given interval of $R b$. For $R b>1$, the within-record variation of the bulk 
Fig. 5 The drag coefficient for $R b>1$ as a function of the within-record variability of the turbulence, $\left(\sigma \overline{w^{\prime} w^{\prime}}\right)^{0.5}$

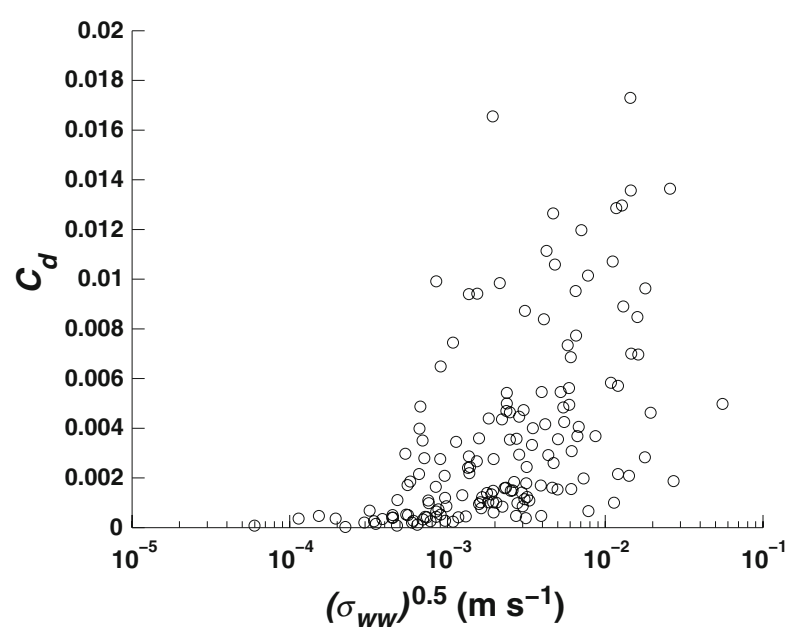

Richardson number is dominated by a variation of the wind speed with a smaller influence of the variation of the temperature stratification. Since the turbulence intensity increases with the submeso velocity scale for $R b>1$ and does not depend significantly on the wind speed, the drag coefficient also increases with increasing submeso velocity scale (not shown), as was observed for $\sigma_{w}$ (Fig. 1).

For $R b>1$, the drag coefficient also increases with the within-record variability of the turbulence as represented by $\left(\sigma \overline{w^{\prime} w^{\prime}}\right){ }^{0.5}$ (Fig. 5) in that the drag coefficient is generally very small for $\left(\sigma \overline{w^{\prime} w^{\prime}}\right)^{0.5}<5 \times 10^{-4} \mathrm{~m} \mathrm{~s}^{-1}$ and then increases with increasing $\left(\sigma \overline{w^{\prime} w^{\prime}}\right){ }^{0.5}$ with large scatter. Records with strong stability can be characterized by significant averaged momentum flux due to intermittent bursts of mixing.

The above influence of the submeso velocity scale on the drag coefficient might be analogous to the decrease of $\phi_{m}$ (greater momentum flux for a given value of wind shear) with an increasing sub-record variation of the Richardson number (Mahrt 2007b). The submeso velocity scale and the sub-record variation of the Richardson number are related (not shown) because the variations of the Richardson number are dominated by variations of the shear. Greater submeso activity leads to a greater within-record variability of the Richardson number, and therefore a greater probability of small Richardson numbers and shear instability somewhere within the record. The dependence of the turbulence on the Richardson number depends on the depth scale and time scale of the calculation. The failure to resolve the local decrease of the Richardson number over thin layers embedded in high Richardson number flow (Balsley et al. 2008) may also contribute to the significant turbulence observed at large values of the Richardson number.

\section{Variability of the Turbulence}

The variability or intermittency of the turbulence within individual records is examined in terms of the within-record scaled standard deviation of the vertical velocity variance (Eq. 7), the skewness of the vertical velocity variance and Rmax (Eq. 6). All three measures are strongly correlated. All three measures of within-record variability increase with decreasing wind speed and increase with increasing bulk Richardson number (not shown). This 
Fig. 6 Upper panel the dependence of the square root of the within-record standard deviation of the vertical velocity variance on the large-scale wind speed, $V$. Lower panel the dependence of the square root of the within-record standard deviation of the vertical velocity variance on the submeso velocity scale, $V_{\text {smeso }}$. Red dots identify cases where the submeso velocity exceeds the large-scale (1-hour) speed $\left(V_{\text {smeso }}>V\right.$ ) while black dots correspond to $\left(V_{\text {smeso }}<V\right)$
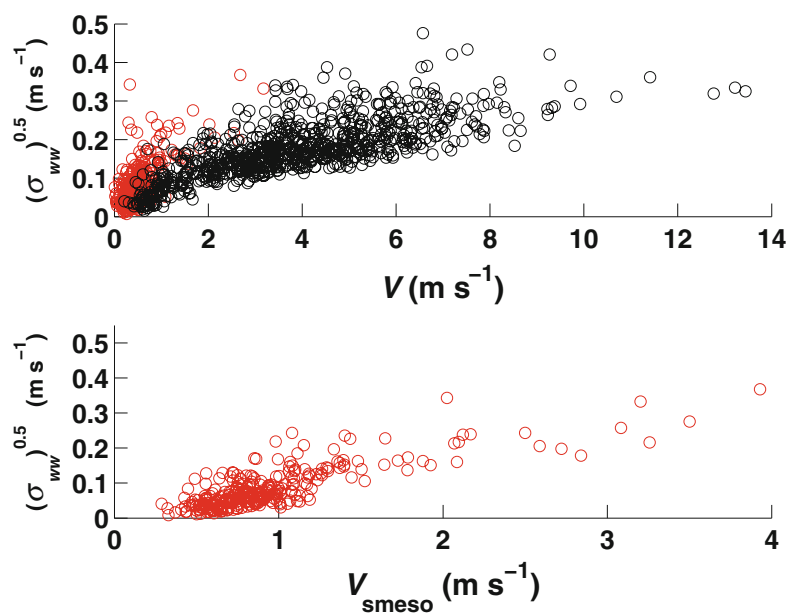

variability of the turbulence is presumably due to, (a) random variability that would occur even in stationary conditions (vanishing submeso motions), and (b) non-stationary submeso motions.

\subsection{Dependence of Variability on Submeso Flow}

We employ the square root of the standard deviation of the vertical velocity variance

$$
\left(\sigma \overline{w^{\prime} w^{\prime}}\right)^{0.5}
$$

as a velocity scale that represents the within-record variability of the turbulence. We will relate this variability velocity scale to $V_{\text {smeso }}$ and the magnitude of the record vector-averaged wind speed at the 2-m level, $V$. Within the large scatter, $V_{\text {smeso }}$ appears to be independent of the wind speed, $V$, except for a possible weak increase for strong winds. Therefore, $V_{\text {submeso }}>V$ occurs primarily for weak winds, less than $2 \mathrm{~m} \mathrm{~s}^{-1}$, consistent with Anfossi et al. (2005). In these cases, the flow is strongly non-stationary with frequent abrupt changes of wind direction (Mahrt 2008).

First, consider the "significant wind regime" where $V>V_{\text {smeso }}$ (black dots, Fig. 6). For this regime, extrapolation of the black dots in the upper panel of Fig. 1 towards vanishing $V$ suggests that the variability of the turbulence, $\left(\sigma_{\overline{w^{\prime} w^{\prime}}}\right)^{0.5}$, vanishes as the large-scale wind speed $(V)$ vanishes. For significant winds, the variability of the turbulence is proportional to the intensity of the turbulence itself, which in turn is proportional to the mean wind speed.

In contrast, the within-record variability of the turbulence, for a given mean wind speed, is significantly larger when the submeso velocity scale becomes important such that $V_{\text {smeso }}>V$ (red dots, Fig. 6, upper panel). For this weak-wind regime, the within-record variability of the turbulence appears to vanish with the vanishing submeso velocity scale (red dots, Fig. 6, lower panel). This behaviour indicates that the within-record variation of the turbulence is more related to submeso motions than "random" variations.

\subsection{Sensitivity to Choice of Variables}

Even though the within-record variability is proportional to the submeso velocity scale, especially for weak large-scale flow (Fig. 6, lower panel), the minute-to-minute variations 
of the turbulence are poorly related to minute-to-minute variations of the submeso motions. Intermittent turbulence observed at a fixed tower is probably generated upwind where the submeso motions are different or in different phase. In theory, the turbulence should be more related to the submeso shear than the submeso velocity. The statistical relationship of $\sigma_{w}$ to submeso variations of the shear is similar to the statistical relationship to the submeso velocity scale. Although the turbulence should be more closely related to the shear than the wind speed, calculation of the shear is more vulnerable to computational errors, particularly for weak winds. Computing submeso variances for the along-wind and crosswind components separately did not improve the prediction of turbulence quantities. Decomposing the flow into different time scales between 1 minute and 1 hour did not reveal a preferred submeso time scale that more effectively generates turbulence. Use of information on the variation of the stratification did not improve the prediction of $\sigma_{w}$.

These analyses are based on only one level near the surface. Based on the seven levels of sonic anemometer data, the velocity aspect ratio increases with height and the intermittency measures decrease with height. The strength of the submeso motions increases slightly with height, but the correlation between the turbulence and submeso activity decreases with height. However, these decreases with height are sufficiently slow that the difference in measurement height between sites is not expected to change the qualitative conclusions of this section.

\section{Between-Site Differences and Intermittency}

The turbulence for very stable conditions is expected to vary significantly between sites, because the surface roughness and the behaviour of the submeso motions vary substantially between sites. The between-site comparisons are only qualitative in that the evaluation of the stability, and selection for the very stable class, are different for each site (Sect. 3.3) due to the different availability of variables and observational levels.

For the very stable regime (Table 1 ), the average value of $\sigma_{w}$ above the canopy is larger for the Pine and Burn sites (Sect. 2) compared to the sites with short vegetation (CASES-99 and FLOSSII). The ratio of the friction velocity to $\sigma_{w}$ is significantly less than one for three of the four sites, but is approximately one for the CASES-99 site. The other three sites all include cases with reversed shear and upward momentum flux. This sign reversal of the momentum flux reduces the value of the friction velocity, which is based on momentum fluxes that are first averaged over all of the very stable records. The cases with shear reversal and an unexpected sign of the momentum flux must be retained to avoid the creation of a bias; the reversed momentum flux is part of the total distribution of values for very stable conditions. Directly averaging $u_{*}$ or eliminating cases with reversed shear would have increased the overall value of $u_{*}$ and made it closer to the averaged value of $\sigma_{w}$. For the FLOSSII data, about $15 \%$ of the records have negative shear due to a weak wind maximum at the ground surface, associated with shallow transient flows (Mahrt 2007b). At the Pine site, the momentum flux above the canopy is upward $22 \%$ of the time, due primarily to a significant sub-canopy drainage flow (Lee and Mahrt 2006) and negative shear above the drainage flow. The Burn site also experiences reversed shear due to a shallow downvalley flow. The negative shear corresponds to severe violation of similarity theory and implies an inflection point in the profile.

The submeso velocity scale averages between 0.6 and $0.8 \mathrm{~m} \mathrm{~s}^{-1}$, except it is only $0.42 \mathrm{~m} \mathrm{~s}^{-1}$ at the Burn site, possibly due to channelling by a narrow valley. $\sigma_{w}$ for very stable conditions is significantly correlated with the submeso velocity scale for the grasscovered CASES-99 and FLOSSII sites, but is less correlated for the two sites with taller 
Table 2 Turbulence variation for $R b>1$

\begin{tabular}{llllllr}
\hline Data & Speed & $R \max$ & $S\left(1 \mathrm{~min} \sigma_{w}\right)$ & $S\left(1 \mathrm{~h} \sigma_{w}\right)$ & r. bet. $\sigma$ & $x /$ along \\
\hline FLOSSII & 0.7 & $6.2(1.9)$ & $2.3(0.5)$ & 2.6 & 1.2 & -0.8 \\
CASES-99 & 1.7 & $5.9(1.9)$ & $2.2(0.1)$ & 1.5 & 0.8 & -3.8 \\
Pine & 1.5 & $4.7(2.4)$ & $1.7(1.3)$ & 4.4 & 1.7 & -0.2 \\
Burn & 1.6 & $4.7(4.5)$ & $2.2(1.9)$ & 10.3 & 1.6 & 0.3 \\
\hline
\end{tabular}

The mean wind speed $\left(\mathrm{m} \mathrm{s}^{-1}\right)$ at the flux level (second column), the average within-record relative maximum of the vertical velocity variance ( $\max$, Eq. 6 with $\phi=w$, values for weak stability are in parentheses), the average within-record skewness of the vertical velocity variance (fourth column), the between-record skewness (fifth column), where $S$ is the skewness, the between-record standard deviation of the vertical velocity variance scaled by the mean vertical velocity variance (rel. $\sigma$, sixth column) and the ratio of the averaged crosswind to along-wind momentum flux (x/along, last column). For the Pine and Burn sites, the data correspond to $z / L>1$ for qualitative comparison

vegetation in complex terrain, perhaps because of the more complex vertical flow structure and interaction with flow within the canopy.

For FLOSSII and CASES-99, the velocity aspect ratio of the turbulence is less than 0.1 while the velocity aspect ratio for the submeso motions is only $10^{-3}$ (Table 1), reflecting the greater restraining influence of the strong stratification and ground surface on the larger-scale submeso vertical motions, which are more two-dimensional near the surface. Above the tall canopies, the velocity aspect ratios are five to ten times larger, presumably because of the lack of a solid boundary at the canopy top; significant vertical velocities are permitted between the tall roughness elements. In conclusion, submeso motions and their impact on turbulence depend on the vegetation and topography of the site.

\subsection{Intermittency Over Short Vegetation}

The maximum 1-min value of the vertical velocity variance, scaled by the record-average value of the vertical velocity variance (third column of Table 2), is large for very stable conditions, about 6 above the short vegetation for $R b>1$. That is, the maximum value of the turbulence within the record is six times larger than the record average. This simple statistic is consistent with the observation that a small fraction of the record accounts for most of the flux in stable intermittent turbulence. This ratio averages only about 2 for weak stability $(0.01<R b<0.1)$. The within-record skewness of the vertical velocity variance (fourth column) is little more than 2 for very stable conditions and an order of magnitude smaller for weak stability, again reflecting the greater intermittency of the turbulence for more stable conditions.

The between-record variability for $R b>1$ is as large as the within-record variation, presumably due to influences not included in the Richardson number. In addition, the chance occurrence of one or two stronger turbulence events within a given hour may lead to appreciable between-record variability. The between-record skewness of the vertical velocity variance is about 2, implying some records with much stronger average turbulence. The betweenrecord relative standard deviation is about 1 , such that the between-record variability of the turbulence is as large as the turbulence itself for very stable conditions.

\subsection{Intermittency Over Tall Vegetation}

Thermodynamic interaction of the stable boundary layer with the low heat capacity vegetation can contribute to the turbulence intermittency (Van de Wiel et al. 2002). For the tall 
vegetation data, the within-record intermittency is larger than for the short vegetation, but these differences depend on stability. For the strong stability class, the within-record intermittency is a little less for the tall vegetation compared to short vegetation (columns 3-4, Table 2). However, the intermittency is not reduced as much for the weak stability class compared to that over short vegetation. The weak stability class is defined as $0.01<z / L<0.1$. Decreasing these stability limits by an order of magnitude to capture even less stable conditions did not qualitatively change the result. Consequently, the intermittency is not as strongly influenced by the stability over the taller vegetation as compared to short vegetation. The two tall vegetation sites are characterized by sparse vegetation and large aerodynamic roughness lengths of more than $1 \mathrm{~m}$ with strongest stratification located within the canopy. Presumably, the intermittency is a function of canopy characteristics as well as stability.

The between-record variation is larger for the tall vegetation. The influences of tall vegetation and complex terrain cannot be clearly separated with the present datasets and they may work in concert. For these two sites, the local drainage flows are confined to the subcanopy, which influences the turbulence above the canopy through directional shear above the drainage flow.

\section{Stress and Wind Directions for $R b>1$}

For weak stability with significant wind, the stress direction is generally opposite to the wind direction. For very stable conditions, the stress direction shows substantial variation relative to the direction of the 1-hr mean wind. For $R b>1$, the crosswind stress averaged over all of the data is positive and averages $75 \%$ of the along-wind stress for FLOSSII (Table 2), as compared to less than $2 \%$ for $R b<1$. Similarly, the shear perpendicular to the mean flow averages $54 \%$ of the along-wind shear and is negative. This clockwise rotation of the stress vector and shear vector at a given level agrees with the rotation of the shear vector resulting from Ekman rotation with height. Similar observations are reported in Grachev et al. (2005). For the present datasets, the influence of baroclinity/drainage flows on the crosswind stress is not known. Average positive crosswind shear and negative crosswind stress also occur for the CASES-99 dataset. The averaged crosswind stress is almost four times as large as the along-wind stress because of a single 2 -hr period of particularly large crosswind stress. This result underscores the large variability of the turbulence for the very stable case and the need for larger datasets. The average crosswind momentum flux is smaller for the Pine and Burn sites and of opposite sign to the Ekman prediction for the Burn site where drainage flows and the narrow valley control the wind direction.

Inclusion of the crosswind momentum flux in the drag law requires parameterization of the stress direction. For simple flow over short vegetation, the above analysis suggests formulation of non-alignment of the stress vector with the wind vector that increases with stability. We reserve such a possible formulation for a future study.

\section{Conclusions}

For very stable conditions, nominally bulk Richardson number $R b>1$, the turbulence is generally weak, with occasional turbulence events. Here, turbulence was defined in terms of the small-scale part of the heat cospectra where the heat transport is systematically gradient (OGIVE method, Sect. 2). Short periods of enhanced turbulent activity can lead to transport that is much greater than the typical transport for strong stability, which even causes 
significant variability of the 1-hr averages. This behaviour corresponds to a large skewness of variations both within records and variations between records for similar mean conditions. Extended periods of extremely weak turbulence of at least one hour may occur, although the turbulence never completely vanishes.

For the very stable regime, the turbulence quantities appear to be independent of the Richardson number and the speed of the large-scale flow, within the large scatter, with no hint of a critical Richardson number. Here, the Richardson number is computed from the large-scale flow (1-hr averages). Some turbulence is generated by the submeso shear, no matter how weak the large-scale flow. As a result, the turbulence intensity and drag coefficient increase with increasing submeso energy. These results suggest that the turbulence never vanishes at large Richardson number partly because of shear generation by the submeso motions.

The important generation of turbulence by the submeso shear for weak-wind conditions leads to unpredictability of the turbulence, since the dynamics of the submeso motions are poorly understood and the submeso motions vary significantly between sites, depending on topography and vegetation, and vary between nights at a given site. As a result, simple universal similarity theory for the turbulence in this regime is unlikely. While the submeso velocity scale explains much of the variation of the turbulence between records for the very stable regime, the remaining variation could be due to other unknown influences, observational errors and the failure of the submeso velocity scale to completely represent the submeso shear generation of turbulence. For weak large-scale wind and significant submeso activity, the within-record variation of the turbulence also increases systematically with increasing strength of the submeso flow.

For the very stable regime, the crosswind stress becomes much more variable and sometimes larger than the along-wind stress. Averaged stress components for the simple sites correspond to a clockwise rotation of the stress vector away from the mean wind. This rotation at a given level is consistent with vertical shear associated with the Ekman rotation of the wind direction with height. For more complex terrain with tall vegetation, the wind-direction change with height can be controlled by directional shear associated with thin drainage flows.

Future progress requires a better understanding of the common melange of submeso motions using time-space information. Formulations of the probability distribution of turbulence quantities would provide practical information, although the probability distribution will be a function of averaging time as well as stability, height above ground and submeso activity.

Acknowledgements The author gratefully acknowledges important comments from Dean Vickers, Carlos Yagüe, Samuel Viana and also valuable comments on the manuscript and future work by the reviewers. The FLOSSII and CASES-99 data were provided by the Integrated Surface Flux Facility of the National Center for Atmospheric Research. Part of this work was carried out as a visiting scientist in the Grup de Meteorologia, Dept. Física, Univ. de les Illes Balears, Palma de Mallorca, Spain under Project CGL2006-12474-CO1 from the Spanish Ministry of Research. This material is based upon work supported by NSF Grant ATM-0607842 and ARO Contract W911FN05C0067.

Open Access This article is distributed under the terms of the Creative Commons Attribution Noncommercial License which permits any noncommercial use, distribution, and reproduction in any medium, provided the original author(s) and source are credited.

\section{References}

Acevedo OC, Moraes OLL, Degrazia GA, Medeiros LE (2006) Intermittency and the exchange of scalars in the nocturnal surface layer. Boundary-Layer Meteorol 119:41-55 
Anderson PS (2003) Fine-scale structure observed in a stable atmospheric boundary layer by Sodar and kite-borne tethersonde. Boundary-Layer Meteorol 107:323-351

Anderson PS (2009) Measurement of Prandtl number as a function of Richardson number avoiding selfcorrelation. Boundary-Layer Meteorol 131:345-362

Andreas E, Geiger C, Treviño G, Claffey K (2008) Identifying nonstationarity in turbulence series. Boundary-Layer Meteorol 127:37-56

Anfossi D, Oettl D, Degrazia G, Goulart A (2005) An analysis of sonic anemometer observations in low wind speed conditions. Boundary-Layer Meteorol 114:179-203

Anquetin A, Guilbaud C, Chollet J-P (1998) The formation and destruction of inversion layers within a deep valley. J Appl Meteorol 37:1547-1560

Balsley B, Svensson G, Tjernström M (2008) On the scale-dependence of the gradient Richardson number in the residual layer. Boundary-Layer Meteorol 127:57-72

Basu S, Porté-Agel F, Foufoula-Georgiou E, Vinuesa J-F, Pahlow M (2006) Revisiting the local scaling hypothesis in stably stratified atmospheric boundary-layer turbulence: an integration of field and laboratory measurements with large-eddy simulations. Boundary-Layer Meteorol 119:473-500

Belušić D, Mahrt L (2008) Estimation of length scales from mesoscale networks. Tellus 60A:706-715

Blackwelder R, Kaplan R (1976) On the wall structure of the turbulent boundary layer. J Fluid Mech 76:89-112

Cava D, Katul G (2009) The effects of thermal stratication on clustering properties of canopy turbulence. Boundary-Layer Meteorol 130:307-325

Coulter R, Doran J (2002) Spatial and temporal occurrence of intermittent turbulence during CASES-99. Boundary-Layer Meteorol 105:329-349

Cuxart J, Morales G, Terradellas E, Yagüe C (2002) Study of coherent structures and estimation of the pressure transport terms for the nocturnal stable boundary layer. Boundary-Layer Meteorol 105:305-328

Derbyshire H (1995) Stable boundary layers: observations, models and variability. Part I: modelling and measurements. Boundary-Layer Meteorol 74:19-54

Doran JC, Horst TW (1981) Velocity and temperature oscillations in drainage winds. J Appl Meteorol 20:360364

Etling D (1990) On plume meandering under stable stratification. Atmos Environ 24A:1979-1985

Friehe C, Shaw W, Rogers D, Davidson K, Large W, Stage S, Crescenti G, Khalsa S, Greenhut G, Li F (1991) Air-sea fluxes and surface layer turbulence around a sea surface temperature front. J Geophys Res 96:8593-8609

Fritts D, Nappo C, Riggin D, Balsley B, Eichinger W, Newsom R (2003) Analysis of ducted motions in the stable nocturnal boundary layer during CASES-99. J Atmos Sci 60:2450-2472

Fritts D, Wang C, Werne J, Lund T, Wan K (2009) Gravity wave instability dynamics at high Reynolds numbers, II: turbulence evolution, structure, and anisotropy. J Atmos Sci 66:1149-1171

Galperin B, Sukoriansky S, Anderson PS (2007) On the critical Richardson number in stably stratified turbulence. Atmos Sci Lett 8:65-69

Grachev AA, Fairall CW, Persson POG, Andreas EL, Guest PS (2005) Stable boundary-layer scaling regimes: the Sheba data. Boundary-Layer Meteorol 116:201-235

Grachev AA, Andreas EL, Fairall CW, Guest PS, Persson POG (2007) On the turbulent Prandtl number in the stable atmospheric boundary layer. Boundary-Layer Meteorol 125:329-341

Hanna SR (1986) Spectra of concentration fluctuations: the two time scales of a meandering plume. Atmos Environ 20:1131-1137

Howell J, Sun J (1999) Surface layer fluxes in stable conditions. Boundary-Layer Meteorol 90:495-520

Katul G, Albertson J, Parlange M, Chu C-R, Stricker H (1994) Conditional sampling, bursting, and the intermittent structure of sensible heat flux. J Geophys Res 99:22869-22876

Lee Y-H, Mahrt L (2006) Effect of stability on mixing in open canopies. Agric For Meteorol 135:169-179

Luhar A, Hurley P, Rayner K (2009) Modelling near-surface low winds over land under stable conditions: sensitivity tests, flux-gradient relationships, and stability parameters. Boundary-Layer Meteorol 130:249274

Mahrt L (2006) Variation of surface air temperature in complex terrain. J Appl Meteorol Clim 45:1481-1493

Mahrt L (2007a) Weak-wind mesoscale meandering in the nocturnal boundary layer. Environ Fluid Mech 7:331-347

Mahrt L (2007b) The influence of transient flow distortion on turbulence in stable weak-wind conditions. Boundary-Layer Meteorol 127:1-16

Mahrt L (2008) Mesoscale wind direction shifts in the stable boundary-layer. Tellus 60A:700-705

Mahrt L (2009) Characteristics of submeso winds in the stable boundary layer. Boundary-Layer Meteorol 130:1-14

Mahrt L, Howell J (1994) The influence of coherent structures and microfronts on scaling laws using global and local transforms. J Fluid Mech 260:247-270 
Mahrt L, Vickers D (2006) Extremely weak mixing in stable conditions. Boundary-Layer Meteorol 119:19-39

Mahrt L, Thomas C, Preuger J (2009) Space-time structure of mesoscale modes in the stable boundary layer. Q J Roy Meteorol Soc 135:67-75

Mestayer PG, Anquetin S (1995) Climatology of cities. In: Gyr A, Rys F-S (eds) Diffusion and transport of pollutants in atmospheric mesoscale flow fields. Kluwer Academic Publishers, Dordrecht, pp 165-189

Muschinski A, Frehlich R, Balsley B (2004) Small-scale and large-scale intermittency in the nocturnal boundary layer and the residual layer. J Fluid Mech 515:319-351

Nakamura R, Mahrt L (2005) A study of intermittent turbulence with CASES-99 tower measurements. Boundary-Layer Meteorol 114:367-387

Nappo CJ (1991) Sporadic breakdown of stability in the PBL over simple and complex terrain. BoundaryLayer Meteorol 54:69-87

Nappo CJ (2002) An introduction to atmospheric gravity waves. Academic Press, New York, $297 \mathrm{pp}$

Ohya Y, Nakamura R, Uchida T (2008) Intermittent bursting of turbulence in a stable boundary layer with low-level jet. Boundary-Layer Meteorol 26:349-363

Panofsky HA, Dutton JA (1984) Atmospheric turbulence-models and methods for engineering applications. Wiley, New York, 397 pp

Poulos G, Blumen W, Fritts D, Lundquist J, Sun J, Burns S, Nappo C, Banta R, Newsom R, Cuxart J, Terradellas E, Balsley B, Jensen M (2002) CASES-99: a comprehensive investigation of the stable nocturnal boundary layer. Bull Am Meteorol Soc 83:555-581

Salmond JA (2005) Wavelet analysis of intermittent turbulence in a very stable nocturnal boundary layer: implications for the vertical mixing of ozone. Boundary-Layer Meteorol 114:463-488

Schwarz P, Law B, Williams M, Irvine J, Kurpius M, Moore D (2004) Climatic versus biotic constraints on carbon and water fluxes in seasonally drought-affected ponderosa pine ecosystems. Glob Biochem Cycles 18:1029-1037

Sorbjan Z, Balsley B (2008) Microstructure of turbulence in the stably stratied boundary layer. BoundaryLayer Meteorol 129:191-210

Sun J, Burns SP, Lenschow DH, Banta R, Newsom R, Coulter R, Frasier S, Ince T, Nappo C, Cuxart J, Blumen W, Lee X, Hu X-Z (2002) Intermittent turbulence associated with a density current passage in the stable boundary layer. Boundary-Layer Meteorol 105:199-219

Sun J, Lenschow DH, Burns SP, Banta RM, Newsom RK, Coulter R, Frasier S, Ince T, Nappo C, Balsley B, Jensen M, Mahrt L, Miller D, Skelly B (2004) Atmospheric disturbances that generate intermittent turbulence in nocturnal boundary layers. Boundary-Layer Meteorol 110:255-279

Terradellas E, Soler M, Ferreres E, Bravo M (2005) Analysis of oscillations in the stable boundary layer using wavelet methods. Boundary-Layer Meteorol 114:489-518

Van de Wiel BJH, Ronda RJ, Moene AF, de Bruin HAR, Holtslag AAM (2002) Intermittent turbulence and oscillations in the stable boundary layer over land. Part I: a bulk model. J Atmos Sci 59:942-958

Van de Wiel BJH, Moene A, Hartogenesis G, De Bruin HAR, Holtsla AAM (2003) Intermittent turbulence in the stable boundary layer over land. Part III: a classification for observations during CASES-99. J Atmos Sci 60:2509-2522

Viana S, Terradellas E, Yagüe C, Maqueda G (2008) Analysis of the different regimes of atmospheric turbulence observed during a single night. Nuovo Cimento C Geophy Space Phys 31:942-958

Vickers D, Mahrt L (2006) A solution for flux contamination by mesoscale motions with very weak turbulence. Boundary-Layer Meteorol 118:431-447

Vickers D, Mahrt L (2007) Observations of the cross-wind velocity variance in the stable boundary layer. Environ Fluid Mech 7:55-71

Vindel JM, Yagüe C, Redondo JM (2008) Structure function analysis and intermittency in the atmospheric boundary layer. Nonlinear Proc Geophy 15:915-929

Yaglom AM (1973) Stationary random functions. Dover, New York, $235 \mathrm{pp}$

Yagüe C, Viana S, Maqueda G, Redondo JM (2006) Influence of stability on the flux-profile relationships for wind speed, $\phi_{m}$, and temperature, $\phi_{h}$, for the stable atmospheric boundary layer. Nonlin Process Geophys 13:185-203

Zilitinkevich SS, Elperin T, Kleeorin N, Rogachevskii I (2007) Energy- and flux-budget (EFB) turbulence closure model for stably stratified flows. Part I: steady state homogenous regimes. Boundary-Layer Meteorol 125:167-191 